\title{
BMJ Open Risk models and scores for metabolic syndrome: systematic review protocol
}

\author{
Musa Saulawa Ibrahim, Dong Pang, Gurch Randhawa, Yannis Pappas
}

To cite: Ibrahim MS, Pang D, Randhawa G, et al. Risk models and scores for metabolic syndrome: systematic review protocol. BMJ Open 2019;9:e027326. doi:10.1136/ bmjopen-2018-027326

- Prepublication history and additional material for this paper are available online. To view these files, please visit the journal online (http://dx.doi. org/10.1136bmjopen-2018027326).

Received 17 October 2018 Revised 26 August 2019 Accepted 02 September 2019

\begin{abstract}
Introduction Metabolic syndrome 'a clustering of risk factors which includes hypertension central obesity, impaired glucose metabolism with insulin resistance and dyslipidaemia' affects approximately $20 \%-25 \%$ of the global adult population. Individuals with metabolic syndrome have two to threefold risk of developing cardiovascular disease and a fivefold risk of developing developing diabetes and death from all causes. Although there is rapid proliferation of risk scores for predicting the risk of developing metabolic syndrome later in life, yet, these are seldom used in the practice. Therefore, the purpose of this review is to determine the performance of risk models and scores for predicting the metabolic syndrome.
\end{abstract}

Methods and analysis Articles will be sought for from electronic databases (MEDLINE, CINAHL, PubMed and Web of Science) as well as the Cochrane Library. Further manual search of reference lists and grey literatures will be conducted. The search will cover from the start of indexing to 3 October 2018. Identified studies will be included if they fulfil the study selection criteria. Quality of studies will be appraised using suitable criteria for the risk models. The risk scores in the final sample of the review will be ranked/prioritised based on previous quality criteria for prognostic risk models. Lastly, the impact of the models will be ascertained by tracking citations on Google Scholar. Ethics and dissemination This study does not require formal ethical approval as primary data will not be collected. The results will be disseminated through a peer-reviewed publication and relevant conference presentations.

PROSPERO registration number CRD42019139326

\section{INTRODUCTION}

Metabolic syndrome (MetS) 'a clustering of risk factors which includes hypertension, central obesity, impaired glucose metabolism with insulin resistance and dyslipidaemia' ${ }^{1}$ is common in most countries of the world. ${ }^{2}$ The global prevalence of MetS is approximately $20 \%-25 \%$ of the adult population ${ }^{2}$ and this is believed to be increasing due to the factors such as ageing population, increase in life expectancy, poor dietary habits, sedentary life style and obesity. ${ }^{3}{ }^{4}$ Furthermore, individuals with MetS have two to threefold risk of developing cardiovascular disease (CVD) and a fivefold risk of developing diabetes (DM)..$^{5}$ They would, therefore, add to the
Strengths and limitations of this study

- This will be a comprehensive review of risk models and scores for predicting metabolic syndrome.

- Will identify good and poor risk models and scores according to the predicting performance of a model.

- Use of methods that are applicable to reviews of any risk models and scores.

- May involve screening large numbers of abstracts at the initial stages due to lack of a sensitive literature search strategy.

- Important additional findings might be missed by limiting the review to only papers published in English.

422.7 million and 230 million people with CVD and DM worldwide, respectively. ${ }^{6}$ It is, therefore, imperative that suitable preventative strategies are adopted in a systematic way to reduce the increasing burden of MetS-related morbidity and mortality.

Furthermore, MetS constitutes a major public health problem, ${ }^{2}$ and therefore, there is a need for urgent development of effective approaches for preventing and managing the syndrome. ${ }^{8}$ This is necessary for proper planning of health services, as well as for building the evidence base required to inform public health policies aimed at reducing the burden of the syndrome and downstream diseases and deaths relating to it.

Risk models simplify reality by estimating the degree to which health or disease is affected through a change in one or more risk factors. ${ }^{9}$ With increasing availability of datasets, computational power and statistical methods, modelling is becoming more popular in chronic disease research. ${ }^{10} 11$ This no doubt has a key role to play in informing how the increasing burden of MetS on population health can be addressed. Recently, there is proliferation of models and scores for assessing risk of developing MetS, however, to the best of our knowledge, none is in routine use either by clinicians or public health practitioners and there is no available systematic review in the academic literature. This may present a confusing picture for both clinicians, 
public health specialists and policy-makers, who would be potentially faced with a very complex literature, multiple different methodologies and probably very few studies of use in real life. Therefore, the purpose of this review is to determine the performance of risk models and scores for predicting MetS.

\section{Research question}

What is the performance of risk models and scores for predicting MetS?

\section{METHODS AND DESIGN \\ Selection criteria}

According to Wakefield, ${ }^{12}$ eligibility criterion is a process of determining the acceptability of a study by carefully defining which studies and samples can be included in a given review. Additionally, setting inclusion criteria provides a framework which enables researchers to limit bias thereby ensuring the validity of the research as well as access to data via process that is strictly accurate. ${ }^{13}$ The inclusion criteria for this review are as follows:

\section{Inclusion}

Study design: this review will include any study deriving or validating a risk model or score for MetS.

Population: as there are studies of MetS risk scores in both children and adults, this study will include studies focusing on any age group.

Intervention/control: this review will focus on studies that develop models or scoring systems based on MetS risk factors to predict temporal risk of MetS and/or validation of an MetS risk model or score.

Outcomes: Any related predictive outcomes (discrimination and calibration inclusive).

\section{Exclusion}

This study will exclude the following:

1. Studies that are still recruiting participants.

2. Studies investigating one or more single risk factors which are not connected to build a model or score.

3. Studies on screening and early detection.

4. Studies of models that predict genetic mutations rather than MetS.

5. Studies conducted on animals.

\section{PATIENT/PUBLIC INVOLVEMENT}

As this study does not involve human subjects, no patients will be involved.

\section{Search strategy}

This study will adopt mixed search strategy which will involve searching both electronic and manual databases in order to identify relevant studies for inclusion. ${ }^{14}{ }^{15}$ The help of the IHR specialist librarian will be sought for while designing the search strategy and the final search strategy will be double checked by authors DP, GR and YP.
The proposed keywords for the literature search include: predict, screen, risk, score, MetS, insulin resistance syndrome, syndrome $\mathrm{X}$, model, regression, risk assessment, risk factor, calculator, analysis, sensitivity and specificity, receiver operating characteristic and OR. Eligible studies will be searched for systematically using synonym free-text words. Also, Truncation commands, Boolean and proximity operators will be used when conducting the searches and adapted in line with the interface used. Search will be limited to English language, but no restriction on publication date will be applied. Samples of the proposed search strategy can be found in online supplementary additional files 1 and 2 .

The literature search will be carried out in databases such as CINAHL, MEDLINE, Web of Science and PubMed. The use of multiple databases minimises selection bias of articles. ${ }^{16}$ Also, MESH search will be performed in the Cochrane Library for MetS and risk. The search will cover from the start of indexing to 3 October 2018.

Additionally, manual search of the reference lists of all the selected articles will be conducted in order to identify more relevant articles. Furthermore, relevant 'grey literature'-research and materials produced by organisations that are not under control of the usual commercial or academic publishers-will be searched for in the following: The Grey Literature Report (http:/ / www.greylit.org/), OpenGrey (www.opengrey.eu/) and OAISTER (http://www.oaister.org/). However, the grey literature search will be limited to abstracts, conferences, relevant stakeholder organisations and reports. Also, to ensure that newly published articles are not missed, Web of Science will be used for forward tracking of previous key published articles. Additionally, PubMed's 'My NCBI' email alert system will be applied using a basic search strategy. Lastly, a complete update search on all the stated databases will be conducted 4 weeks before the final submission of the review draft. In the event that new articles fit for inclusion are detected, they will be evaluated and the findings incorporated in the review before the final submission.

Finally, search results from the various sources will be imported into Endnote V.8 (endnote.com) and merged. Thereafter, duplicates will be screened and removed both electronically and manually.

\section{Selection of studies}

After identification of articles from searching electronic databases, one author (MSI) will conduct screening of their titles and abstracts according to the eligibility criteria. Subsequently, a second review author (DP) will conduct a check on a random sample of $10 \%$ of those screened articles. Furthermore, the full text papers of all the potentially eligible studies will be obtained (including those identified via non-electronic means). Review author (MSI) will then apply the full inclusion criteria to the full text articles and in event of any confusion/difficulty; this will be discussed with review authors (DP) (GR) and (YP). The review will be carried out using 'Preferred Reporting 
Items for Systematic Reviews and Meta-Analyses Protocols (PRISMA) checklist, ${ }^{17}$ PRISMA flow diagram will be used to show the selection process and Endnote V.8 (endnote. com) will be used for managing references.

\section{Quality assessment}

The Prediction study Risk Of Bias Assessment Tool ${ }^{18}$, a tool for assessing the risk of bias and applicability of prognostic model studies, will be used by review author (MSI) to appraise the individual studies. Difficulties encountered will be discussed and resolved with review authors (DP, GR and YP).

\section{Data extraction and management}

Review author (MSI) will extract data using a standard form adopted from a similar study ${ }^{19}$ (see online additional file 3) and saved in Microsoft Excel 2016. Data to be extracted will be on the details specific to the review question and that satisfies the conditions for the narrative synthesis. The extracted data will be double checked by review authors (DP, GR and YP) and all conflicts will be resolved.

\section{ANALYSIS}

\section{Data synthesis (data analysis plan)}

Although similar metabolic risk factors are used in defining MetS criteria, yet, they differ in terms of the cut-off levels of the basic components, the emphasis given to particular components or the pathophysiological basis underpinning the clustering. ${ }^{20}$ However, this lack of universally acceptable definition makes it difficult to compare different studies. Therefore, in this review, studies reporting prognostic models will be summarised using narrative synthesis due to anticipated heterogeneity of data across the studies (this potentially precludes a formal meta-analysis). The narrative synthesis will first report the number of studies to be included in the review, and then describe the characteristics of those studies including the study population and location. Subsequently, the statistical properties of the models-what variables (prognostic factors) were included in the final model; how the included variables were coded; what the specification of the model was and how it produces an individual outcome probability or risk score; the reported predictive accuracy of the model (reported measures of discrimination and calibration); and whether the model was validated internally and/or externally, and if so, how?. Finally, the narrative synthesis will report the observed similarities and differences of findings, reflect on strength of findings and evaluate between study heterogeneity. All data analyses will be conducted separately for children and adults.

The risk scores in the final sample of the review will be ranked/prioritised based on previous quality criteria for prognostic risk models. ${ }^{21}$ Studies will be favoured based on their generalisability (externally validated by a separate research team on a different population) and usability (calibration that is statistically significant, and discrimination above 0.70$)$.

\section{IMPACT ANALYSIS}

The impact of each prediction model in the final sample will be assessed based on the following: 'any description in the paper of use of the score beyond the population for whom it was developed and validated; number of citations of the paper in Google Scholar and number of these that described use of the score in an impact study; and critical appraisal of any impact studies identified on this citation track'. ${ }^{19}$ The guiding question of this phase of the review is: what is the evidence that this risk score has been used in an intervention which improved (or sought to improve) outcomes for individuals at high risk of MetS?

\section{CONCLUSION}

This systematic review will be the first to assess the performance of existing risk scores for predicting MetS. It is expected that the findings of this review will be an important step towards informing the choice of potential MetS risk score both in clinical and public health decision-making. Particularly, the review will identify the various MetS risk scores currently in existence and examine their methodological strengths. Furthermore, the impact that the individual models had to practise so far will be investigated. Lastly, the identified risk scores will be ranked using standardised procedure in order to aid their selection by potential users.

\section{Study status}

The study is ongoing and is expected to be completed by September 2019.

Acknowledgements The authors will like to acknowledge the contribution of David Abdy, the specialist librarian for Institute for Health Research, University of Bedfordshire for his contribution in designing the search strategy for the study.

Contributors MSI drafted the protocol, search strategy and manuscript. DP, GR and YP read, provided feedback and approved the final manuscript.

Funding The authors have not declared a specific grant for this research from any funding agency in the public, commercial or not-for-profit sectors.

Competing interests None declared.

Patient consent for publication Not required.

Ethics approval This study does not require formal ethical approval as primary data will not be collected. The results will be disseminated through a peer-reviewed publication and relevant conference presentations.

Provenance and peer review Not commissioned; externally peer reviewed.

Open access This is an open access article distributed in accordance with the Creative Commons Attribution Non Commercial (CC BY-NC 4.0) license, which permits others to distribute, remix, adapt, build upon this work non-commercially, and license their derivative works on different terms, provided the original work is properly cited, appropriate credit is given, any changes made indicated, and the use is non-commercial. See: http://creativecommons.org/licenses/by-nc/4.0/.

\section{REFERENCES}

1. Expert Panel on Detection, Evaluation, and Treatment of High Blood Cholesterol in Adults. Executive summary of the third report of the National cholesterol education program (NCEP) expert panel on detection, evaluation, and treatment of high blood cholesterol in adults (adult treatment panel III). JAMA 2001;285:2486-97.

2. International Diabetes Federation. IDF Diabetes Atlas Eighth Edition 2017, 2017. Available: file://C:/Users/1514026/Downloads/IDF_ Atlas_7e_2015_EN.pdf [Accessed 20 May 2018]. 
3. Xavier HT, Monte O. Atherosclerosis prevention in metabolic syndrome patients: from physiopathology to the farmacoeconomics of statins treatment. Rev Bras Med 2005;62:197-204.

4. Hossain P, Kawar B, El Nahas M. Obesity and diabetes in the developing world - a growing challenge. N Engl J Med Overseas Ed 2007;356:213-5.

5. Ahrens W, Moreno LA, Mårild S, et al. Metabolic syndrome in young children: definitions and results of the IDEFICS study. Int $J$ Obes 2014;38:S4-S14.

6. International Diabetes Federation. IDF Diabetes Atlas Eighth Edition 2017, 2017. Available: file:///C:/Users/1514026/Downloads/IDF_DA_ 8e-EN-final.pdf [Accessed : 20 May 2018].

7. Roth GA, Johnson C, Abajobir A, et al. Global, regional, and national burden of cardiovascular diseases for 10 causes, 1990 to 2015. J Am Coll Cardiol 2017;70:1-25.

8. Grundy SM. Metabolic syndrome pandemic. Arterioscler Thromb Vasc Biol 2008;28:629-36.

9. Webber L, Mytton OT, Briggs ADM, et al. The Brighton declaration: the value of non-communicable disease modelling in population health sciences. Eur J Epidemiol 2014;29:867-70.

10. Maglio PP, Mabry PL. Agent-based models and systems science approaches to public health. Am J Prev Med 2011;40:392-4.

11. Briggs AScarborough PSmith A20166790

12. Wakefield A. Searching and critiquing the research literature. Nursing Standard 2014;28:49-57.
13. Evidence for Policy and Practice Information and Co-ordinating Centre. EPPICentre methods for conducting systematic reviews, 2010. Available: http://eppi.ioe.ac.uk/cms/LinkClick.aspx?fileticket= hQBu8y4uVwl\%3D [Accessed : 02 May 2018].

14. Brereton $P$, Kitchenham BA, Budgen D, et al. Using a protocol template for case study planning. InEASE 2008:41-8.

15. Bettany-Saltikov J. How to do a systematic literature review in nursing: a step-by-step guide. McGraw-Hill Education (UK), 2012.

16. Relevo R, Balshem $\mathrm{H}$. Finding evidence for comparing medical interventions: $A H R Q$ and the effective health care program. J Clin Epidemiol 2011;64:1168-77.

17. Moher Det al. Preferred reporting items for systematic reviews and meta-analyses: the PRISMA statement. Ann Intern Med 2009;151:264-9.

18. Wolff RF, Moons KGM, Riley RD, et al. PROBAST: a tool to assess the risk of bias and applicability of prediction model studies. Ann Intern Med 2019;170:51-8.

19. Noble D, Mathur R, Dent T, et al. Risk models and scores for type 2 diabetes: systematic review. BMJ 2011;343:d7163.

20. Balkau $B$, Valensi $P$, Eschwège $E$, et al. A review of the metabolic syndrome. Diabetes Metab 2007;33:405-13.

21. Altman DG, Vergouwe $Y$, Royston $P$, et al. Prognosis and prognostic research: validating a prognostic model. BMJ 2009;338:b605. 